
\title{
Research Suare \\ Performance Analysis of Reconfigurable Intelligent Surface in a Dual-Hop DF Relay Empowered Asymmetric RF/FSO Networks
}

Kehinde 0 Odeyemi ( $\sim$ kesonics@yahoo.com )

Tshwane University of Technology - Shoshanguve Campus https://orcid.org/0000-0002-8104-9118

Pius A. Owolawi

Tshwane University of Technology - Shoshanguve Campus

Oladayo 0. Olakanmi

University of Ibadan Faculty of Technology

\section{Research Article}

Keywords: Reconfigurable intelligent surface, decode-and-forward relay, radio frequency, free space optical, atmospheric turbulence, pointing errors

Posted Date: June 17th, 2021

DOl: https://doi.org/10.21203/rs.3.rs-610003/v1

License: (c) (i) This work is licensed under a Creative Commons Attribution 4.0 International License. Read Full License 


\title{
Performance Analysis of Reconfigurable Intelligent Surface in a Dual-Hop DF Relay Empowered Asymmetric RF/FSO Networks
}

\author{
Kehinde O. Odeyemi ${ }^{1,2 *}$, Pius A. Owolawi ${ }^{1}$ and Oladayo O. Olakanmi ${ }^{2}$ \\ ${ }^{1}$ Department of Computer Systems Engineering, Tshwane University of Technology, \\ Pretoria-0001, South Africa \\ ${ }^{2}$ Department of Electrical and Electronic Engineering, Faculty of Technology, University of \\ Ibadan, Nigeria \\ 1kesonics@yahoo.com, ${ }^{1}$ p.owolawi@gmail.com, ${ }^{2}$ olarad4@yahoo.com
}

\begin{abstract}
In this paper, the performance analysis of a reconfigurable intelligent surface (RIS) in a dualhop decode-and-forward (DF) relay empowered asymmetric radio frequency (RF)/free space optical (FSO) systems is presented. The RIS-assisted RF network is subjected to Nakagami-m distribution while the RIS-assisted FSO networks experience Gamma-Gamma distribution in which both atmospheric turbulence and pointing errors are considered. Thus, the closed-form expressions for the system outage probability and average bit error rate (ABER) are derived with limited number of reflecting elements at RIS-assisted RF network and multiple number of reflecting elements at RIS-assisted FSO network. Further, to obtain more insight about the system characteristic, the asymptotic closed-form expressions are derived at high signal-tonoise ratio (SNR) for the system performance metrics. The results illustrate the impact of the system and channel parameters on the proposed system in terms of atmospheric turbulence, pointing errors under beam width condition, $m$-fading parameter, and number of reflecting elements. The correctness of the derived analytical expressions is validated via the MonteCarlo simulations.
\end{abstract}

Keywords: Reconfigurable intelligent surface, decode-and-forward relay, radio frequency, free space optical, atmospheric turbulence, pointing errors

\section{Introduction}

\section{a) Background Information}

Transmission of information from source to the destination can mostly be achieved through either the radio frequency (RF) or optical technologies. Actually, the RF-based wireless system provides wide operational range but suffers from few challenges such as scarcity of spectrum, interference, lower capacity and so on [1]. As a result of this, free space optical (FSO) has been considered as a promising solution owing to its advantages that includes unlicensed spectrum, higher data rate, low cost and easy of deployment, immunity to inference etc [2]. However, the performance of FSO system is highly degraded by atmospheric turbulence induce-fading causing fluctuation in the refractive index due to inhomogeneities in temperature and pressure of the environment [3]. As a line-of-sight system, it is susceptible to pointing error effects as a result of building sway leading to misalignments between the system transmitter and receiver [4].

Cooperative relaying technology has been widely employed in wireless communication system 
to prevent the possibility of the entire system from experiencing deep fading and make system more robust. Consequently, the technology causes the system to has an asymmetric fading distribution in which RF transmission can be employed at one hop and FSO transmission at the other in a dual-hop configuration [5, 6]. This provides 'last-mile' connection and also fill the connectivity gap between the RF and FSO backbone network [2, 7]. As a result of this, an asymmetric RF/FSO relaying system combines the advantages of both the RF and FSO communication technologies to establish a wireless network having wide coverage area and bandwidth [8,9]. These attractive features of asymmetric RF/FSO relaying systems make them a strong candidate for current and future wireless networks. Therefore, huge research studies have investigated the performance of asymmetric RF/FSO relaying system under different performance indices.

Recently, reconfigurable intelligent surface (RIS) has been regarded as a hopeful candidate for the future wireless communication. As an artificial surface, it composes of electromagnetic materials that is capable of controlling the signal propagation electronically through the integrated electronics [10]. Based on this, it has potential to efficiently customize the wireless environment in order to realize wireless system with high spectrum and energy efficiencies [11]. Compare to other conventional technologies, it can intelligently controls the incident signal characteristics such as amplitude, phase, frequency without the need of communication operation processes $[12,13]$. This eliminates the dead zone issues experience in RF networks by creating smart communication channel and environment or as a wave-guide for optical communication [14].

\section{b) Literature and Motivation}

Due to the fact that cooperative relaying technique offers wide operation range and established reliable communication under heavy channel environments and huge obstacles, RIS has been recently extended to cooperative transmission. The RIS research topics have been widely studied in open literature, specifically, in [12] where the performance of RIS-empowered UAV relaying network was evaluated. Also, the performance of a RIS-assisted network over Nakagami-m was investigated in [11]. Moreover, the authors in [15] studied the performance of RIS-assisted internet-of-things system over $F$-distribution induced fading. The influence of modulation index scheme on the RIS-based space shift key wireless system was studied in [16]. Odeyemi et al [17] investigated the performance of RIS-assisted power line communication network under different relay protocols. Further, the authors in [18, 19] evaluated the performance of RIS-enhanced underwater communication systems. The physical layer security performance of a vehicle network with RIS was evaluated in [20,21]. In addition, the impact of co-channel interference on RIS-based system was investigated in [22]. Also, authors in [23] studied the RIS performance in wireless-powered interference-limited communication network. The performance of RIS-enhance dual-hop FSO system was presented in [13] under the influence atmospheric turbulence and pointing error. However, all the aforementioned works on the RIS cooperative relaying are not asymmetric based cooperative system. Thus, the performance of RIS in the mixed RF/FSO relaying system was evaluated in [24] with opportunistic user scheduling. Further, authors in [25] presented a dualhop mixed RF/FSO communication system through RIS. In all this stated works however, the RIS was only used to assist the RF link to propagate information and was not considered for the FSO link. Motivated by this, this paper presented the performance of a RIS in a dual-hop decode-and-forward relay empowered asymmetric RF/FSO systems. Therefore, it is assumed 
that there are huge obstacles between the source-to-relay and relay-to-destination links and RISs are utilized as means of transmission on both hops. Under the atmospheric turbulence and pointing errors, the closed-form expressions for the system outage probability and average error rate are derived. In order to obtain further insight about the derived analytical expressions, the asymptotic closed-form expressions are obtained for the concerned system at high SNR. Thus, the main contributions of this study are summarised as follows:

i. It is assumed that there are huge obstacles between the source-to-relay and relay-todestination links, thus the closed-form expression for the system outage probability is derived

ii. The analytical expression for the concerned system average bit error rate is obtained

iii. The asymptotic expression for the system outage probability and ABER are obtained at high SNR

iv. Differing from [24] and [25] where RIS was used to enhance RF link, in this work, the RISs are utilised in the proposed system to assist both the RF and FSO link.

The rest of the paper is organized as follows. In Section II, the system model and statistical channel characteristics are provided. The system exact analytical expressions with asymptotic expressions of the outage probability and ABER are derived in Section III. Numerical results and discussions are detailed in Section IV and finally, concluding remarks are given in Section V.

\section{System and Channel models}

A dual-hop asymmetric RF/FSO network where the source (S) communicates with destination (D) via the RIS and DF-based relay node is demonstrated in figure 1. The source is equipped with a single transmit antenna, the relay node is provided with a single receive antenna and a single transmit photo-aperture, the destination is furnished with a single receive photo-detector while the RISs are equipped with $N$ number of reflecting elements. The RIS-RF links are assumed to be Nakagami-m distributions and the RIS-FSO is subjected to Gamma-Gamma distributions with pointing errors. Owing to obstruction between the source-to-relay and relayto-destination, the communication is established through the RIS located on buildings to offer connectivity between the source and the destination. As a cooperative-based network, the overall transmission occurs in two phases and the network operates in half-duplex mode. During the first phase, the source sends information to RIS-RF which is reflected to relay node. The relay node the converts the reflected RF signal to an optical signal and finally forward the source information to the RIS-FSO which also reflect the optical signal to the destination.

In DF relaying scheme, the end-to-end signal-to-noise ratio (SNR) can be expressed as [24, 26]:

$$
\gamma=\frac{\gamma_{R F} \gamma_{F S O}}{\gamma_{R F}+\gamma_{F S O}+1} \approx \min \left(\gamma_{R F}, \gamma_{F S O}\right)
$$

where $\gamma_{1}$ represents the instantaneous SNR of the RF link and can be expressed as [11]:

$$
\gamma_{R F}=\frac{P_{S}}{N_{o}}\left(\sum_{p=1}^{N_{R F}} u_{p} v_{p}\right)^{2}=\bar{\gamma}_{R F} Z^{2}
$$


where the $P_{R F}$ is the transmit power used by the source, $N_{R F}$ is the number of reflecting elements on the RIS-RF, $u_{p}$ and $v_{p}$ are the channels amplitude which are independent random variable of the RF link, and the $\bar{\gamma}_{R F}=P_{S} / N_{o}$ is the average SNR of the RF link with $N_{o}$ signifies the noise power that is assumed to be additive white Gaussian noise with zero mean. The $\gamma_{2}$ denotes the instantaneous SNR of the FSO link which can be given as [13]:

$$
\gamma_{F S O}=\frac{P_{R}}{N_{o}} \sum_{q=1}^{N_{F S O}} h_{q}^{2}=\bar{\gamma}_{F S O} W
$$

where $P_{R}$ is the relay transmit power, $N_{F S O}$ is the number of reflecting elements on the RISFSO, $h_{q}$ is the $q-t h$ channel gain of the FSO link and $\bar{\gamma}_{F S O}=P_{R} / N_{o}$

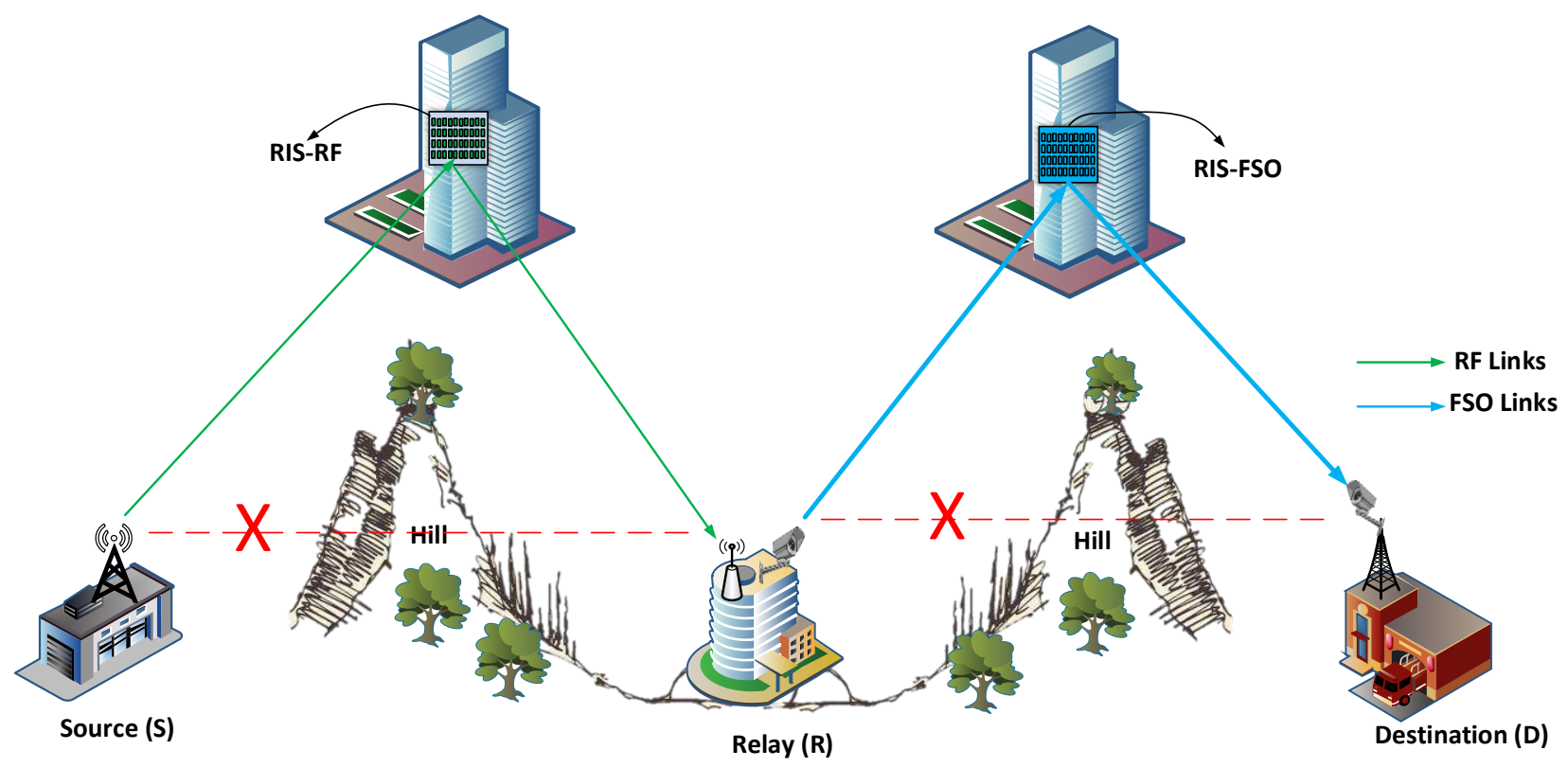

Figure 1: System model of RIS in asymmetry RF/FSO network

In this paper, it is assumed that the RF link follows Nakagami-m distributions. Thus, the probability distribution function (PDF) of the instantaneous SNR for the product of two Nakagami-m random variables can be expressed as [11, 20]:

$$
f_{R F}(\gamma) \approx \frac{\exp \left(\gamma^{1 / 2} / \sqrt{\bar{\gamma}_{R F} \rho^{2}}\right)}{2 \rho^{\lambda+1} \Gamma(\lambda+1) \sqrt{\bar{\gamma}_{R F}}} \gamma^{\frac{\lambda+1}{2}}
$$

where

$$
\left\{\begin{array}{c}
\lambda=\frac{\left(N_{R F}+1\right) \Gamma(m+1 / 2)^{4}-m^{2} \Gamma(m)^{4}}{m^{2} \Gamma(m)^{4}-\Gamma(m+1 / 2)^{4}} \\
\rho=\frac{m \Omega\left(\Gamma(m)^{2} \Gamma(m+1)^{2}-\Gamma(m+1 / 2)^{4}\right)}{\Gamma(m+1 / 2)^{2} \Gamma(m+1)^{2}}
\end{array}\right.
$$

and the cumulative distribution function $(\mathrm{CDF})$ can be defined as: 


$$
F_{R F}(\gamma) \approx \gamma\left(\lambda+1, \frac{\gamma^{1 / 2}}{\sqrt{\bar{\gamma}_{R F} \rho^{2}}}\right)
$$

where $\gamma(.,$.$) incomplete Gamma function$

By converting the lower incomplete Gamma function in (6) to Meijer-G function using the identity detailed in [27, Eq.(8.4.16(1))], the RF CDF can be expressed as:

$$
F_{R F}(\gamma) \approx \frac{1}{\Gamma(\lambda+1)} G_{1,2}^{1,1}\left(\frac{\gamma^{1 / 2}}{\sqrt{\bar{\gamma}_{R F} \rho^{2}}} \mid \begin{array}{c}
1 \\
\lambda+1,0
\end{array}\right)
$$

Similarly, it is assumed that the FSO link undergoes Gamma-Gamma distribution with pointing error. Thus, the pointing error occurs on the RIS-FSO due to beam jitter and intelligent channel reconfigurable node (ICRN) jitter. According to [28], the superimposed pointing error angle $\theta_{k}^{S}$ between the ICRN reflection point and the actual incident point at the receiver can be modelled by a Rayleigh distribution. Therefore, the PDF of the instantaneous SNR with pointing error can be obtained as [13]:

$$
f_{F S O}(\gamma)=\frac{\alpha \beta \xi}{2 \sqrt{\gamma \bar{\gamma}_{F S O}} \Gamma(\alpha) \Gamma(\beta) A_{o}} G_{1,3}^{3,0}\left(\left.\frac{\alpha \beta}{A_{o}} \sqrt{\frac{\gamma}{\bar{\gamma}_{F S O}}}\right|_{\xi-1, \alpha-1, \beta-1}\right)
$$

where $\xi=\frac{\omega_{e q}^{2}}{4 \sigma_{\theta}^{2} D_{T}+16 \sigma_{\beta}^{2} D_{2}^{2}}$ with $\omega_{e q}^{2}=\frac{\omega_{Z}^{2} \sqrt{\pi} \operatorname{erf}(v)}{2 \exp \left(-v^{2}\right)}, A_{o}=\operatorname{erf}^{2}(v), v=\sqrt{\pi / 2} a / w_{z}$ which is the ratio of aperture radius $a$ and beam width $w_{z}$. The beam width $w_{z}$ can be defined as $w_{z}=\phi D_{T}$ with $\phi$ denotes as the divergent angle of the beam width and $D_{T}=D_{1}+D_{2}$ with $D_{1}$ and $D_{2}$ respectively represent the distance between relay and the RIS, and between the RIS and destination. The $\sigma_{\theta}^{2}$ and $\sigma_{\beta}^{2}$ signify the variance of pointing error angle and deflection error angle for ICRN respectively. Thus, the mean and variance of the PDF given in (8) can be expressed as:

$$
\left\{\begin{array}{c}
\mu_{1}=\frac{\xi A_{o}^{2} \Gamma(\xi+2) \Gamma(\alpha+2) \Gamma(\beta+2)}{\alpha^{2} \beta^{2} \Gamma(\alpha) \Gamma(\beta) \Gamma(\xi+3)} \\
\sigma_{1}^{2}=\frac{\xi A_{o}^{4} \Gamma(\xi+4) \Gamma(\alpha+4) \Gamma(\beta+4)}{\alpha^{4} \beta^{4} \Gamma(\alpha) \Gamma(\beta) \Gamma(\xi+5)}-\mu_{1}^{2}
\end{array}\right.
$$

By assuming large $N_{F S O}$ at the RIS-FSO, the (8) can be well approximated using central limit theorem detailed in [29] with $\sigma^{2}=N_{F S O} / \sigma_{1}^{2}$ and $\mu=\mu_{1} \times N_{F S O}$. The PDF given in (8) can thus be expressed as:

$$
f_{F S O}(\gamma) \approx \frac{1}{\sqrt{2 \pi \sigma^{2} \bar{\gamma}_{F S O}^{2}}} \exp -\left(\frac{\gamma-\bar{\gamma}_{F S O} \mu}{2 \sigma^{2} \bar{\gamma}_{F S O}^{2}}\right)
$$

For easy simplification, the exponential function in (10) is then converted into infinite series form using the identity defined in [30, Eq.(1.211(3))]:

$$
f_{F S O}(\gamma) \approx \frac{1}{\sqrt{2 \pi \sigma^{2} \bar{\gamma}_{F S O}^{2}}} \sum_{k=0}^{\infty} \frac{(-1)^{k}}{k !\left(2 \sigma^{2} \bar{\gamma}_{F S O}^{2}\right)^{k}}\left(\gamma-\bar{\gamma}_{F S O} \mu\right)^{2 k}
$$


By using binomial expansion for $\left(\gamma-\bar{\gamma}_{F S O} \mu\right)^{2 k}$ detailed in [30], the (11) can be further expressed as:

$$
f_{F S O}(\gamma) \approx \frac{1}{\sqrt{2 \pi \sigma^{2} \bar{\gamma}_{F S O}^{2}}} \sum_{k=0}^{\infty} \sum_{j=0}^{2 k}\left(\begin{array}{c}
2 k \\
j
\end{array}\right) \frac{(-1)^{k+j}\left(\bar{\gamma}_{F S O} \mu\right)^{j}}{k !\left(2 \sigma^{2} \bar{\gamma}_{F S O}^{2}\right)^{k}} \gamma^{2 k-j}
$$

The CDF of the RIS-FSO can be obtained by integrating (12) as follows:

$$
F_{F S O}(\gamma) \approx \frac{1}{\sqrt{2 \pi \sigma^{2} \bar{\gamma}_{F S O}^{2}}} \sum_{k=0}^{\infty} \sum_{j=0}^{2 k}\left(\begin{array}{c}
2 k \\
j
\end{array}\right) \frac{(-1)^{k+j}\left(\bar{\gamma}_{F S O} \mu\right)^{j}}{k !\left(2 \sigma^{2} \bar{\gamma}_{F S O}^{2}\right)^{k}(2 k-j+1)} \gamma^{2 k-j+1}
$$

\section{Performance Analysis}

In this study, the outage probability and the average error rate are the performance metrics considered for the concerned systems.

\section{a) Statistical Characteristics}

The equivalent end-to-end instantaneous SNR for the concerned system under the DF relaying protocol can be expressed as [17]:

$$
F_{e q}(\gamma)=\operatorname{Pr}\left(\min \left(\gamma_{R F}, \gamma_{F S O}\right)<\gamma\right)
$$

This can be further expressed as:

$$
F_{e q}(\gamma)=F_{R F}(\gamma)+F_{F S O}(\gamma)-F_{F S O}(\gamma) F_{R F}(\gamma)
$$

where $F_{R F}(\gamma)$ and $F_{F S O}(\gamma)$ are the CDF of the RF and FSO respectively

By putting (7) and (13) into (15), the system outage probability can be obtained as:

$$
\begin{gathered}
F_{e q}\left(\gamma_{t h}\right)=\frac{1}{\Gamma(\lambda+1)} G_{1,2}^{1,1}\left(\frac{\gamma^{1 / 2}}{\sqrt{\bar{\gamma}_{R F} \rho^{2}}} \mid \begin{array}{c}
1 \\
\lambda+1,0
\end{array}\right) \\
+\frac{1}{\sqrt{2 \pi \sigma^{2} \bar{\gamma}_{F S O}^{2}}} \sum_{k=0}^{\infty} \sum_{j=0}^{2 k}\left(\begin{array}{c}
2 k \\
j
\end{array}\right) \frac{(-1)^{k+j}\left(\bar{\gamma}_{F S O} \mu\right)^{j}}{k !\left(2 \sigma^{2} \bar{\gamma}_{F S O}^{2}\right)^{k}(2 k-j+1)} \gamma^{2 k-j+1} \\
-\frac{1}{\Gamma(\lambda+1) \sqrt{2 \pi \sigma^{2} \bar{\gamma}_{F S O}^{2}}} \sum_{k=0}^{\infty} \sum_{j=0}^{2 k}\left(\begin{array}{c}
2 k \\
j
\end{array}\right) \frac{(-1)^{k+j}\left(\bar{\gamma}_{F S O} \mu\right)^{j}}{k !\left(2 \sigma^{2} \bar{\gamma}_{F S O}^{2}\right)^{k}(2 k-j+1)} \gamma^{2 k-j+1} G_{1,2}^{1,1}\left(\frac{\gamma^{1 / 2}}{\sqrt{\bar{\gamma}_{R F} \rho^{2}}} \mid \begin{array}{c}
1 \\
\lambda+1,0
\end{array}\right)
\end{gathered}
$$

\section{b) Outage Probability}

Outage probability is a vital index in quantifying the performance of a wireless system. Thus, the system outage occurs when the instantaneous SNR $\gamma$ falls below a predefined threshold $\gamma_{t h}$. The outage probability of the concerned system can therefore be expressed as [22]:

$$
P_{\text {out }}\left(\gamma_{t h}\right)=F_{e q}\left(\gamma_{t h}\right)
$$

Thus, the system outage probability cab then be obtained by invoking (16) into (17). 


\section{Asymptotic Outage Probability}

In the outage probability expression derived in (16), it is very difficult to study the impact of model parameters on the system performance owing to its complexity. Thus, obtaining asymptotic expression from (16) will help in providing more insight about the system behaviour at high SNR region. Neglecting the last term of (15) gives an accurate end-to-end equivalent $\mathrm{CDF}$ at high SNR and can be expressed as [31]:

$$
F_{\text {out }}^{\infty}\left(\gamma_{t h}\right) \approx F_{R F}(\gamma)+F_{F S O}(\gamma)
$$

Obviously, as the $\bar{\gamma}_{R F} \rightarrow \infty$, the $F_{R F}(\gamma)$ can be simplified by obtaining the asymptotic series expansion of Meijer-G function through the identity detailed in [19, 30]. Thus, the system endto-end CDF at high SNR can be written as:

$$
\begin{aligned}
F_{\text {out }}^{\infty}(\gamma) \approx & \frac{1}{\Gamma(\lambda+2)}\left(\frac{\gamma^{1 / 2}}{\sqrt{\bar{\gamma}_{R F} \rho^{2}}}\right)^{\lambda+1} \\
& \quad+\frac{1}{\sqrt{2 \pi \sigma^{2} \bar{\gamma}_{F S O}^{2}}} \sum_{k=0}^{\infty} \sum_{j=0}^{2 k}\left(\begin{array}{c}
2 k \\
j
\end{array}\right) \frac{(-1)^{k+j}\left(\bar{\gamma}_{F S O} \mu\right)^{j}}{k !\left(2 \sigma^{2} \bar{\gamma}_{F S O}^{2}\right)^{k}(2 k-j+1)} \gamma^{2 k-j+1}
\end{aligned}
$$

Thus, the outage probability at high SNR can be determined by putting (19) into (17).

\section{c) Average Bit Error Rate (ABER)}

The average error rate of the concerned network can be obtained as follows [32]:

$$
A B E R=\frac{q^{p}}{2 \Gamma(p)} \int_{0}^{\infty} \exp (-q \gamma) F_{e q}(\gamma) d \gamma
$$

where $p$ and $q$ are the modulation parameters which indicate the type of modulation scheme. For example, when $p=1 / 2$ and $q=1$ denote binary phase shift keying (BPSK), $p=1$ and $q=1$ signify differential phase shift keying (DPSK).

By invoking (16) into (20), the error of the system can be expressed as:

$$
A B E R=\frac{q^{p}}{2 \Gamma(p)}\left[P_{b_{1}}+P_{b_{2}}-P_{b_{3}}\right]
$$

where

$$
P_{b_{1}}=\frac{1}{\Gamma(\lambda+1)} \int_{0}^{\infty} \gamma^{p-1} \exp (-q \gamma) G_{1,2}^{1,1}\left(\frac{\gamma^{1 / 2}}{\sqrt{\bar{\gamma}_{R F} \rho^{2}}} \mid \begin{array}{c}
1 \\
\lambda+1,0
\end{array}\right) d \gamma
$$

For easy simplification, the exponential function can be converted to Meijer-G function using the identity detailed in [33, Eq.(11)]. Then, the (22) can be expressed as:

$$
P_{b_{1}}=\frac{1}{\Gamma(\lambda+1)} \int_{0}^{\infty} \gamma^{p-1} G_{0,1}^{1,0}\left(\left.q \gamma\right|_{0} ^{-}\right) G_{1,2}^{1,1}\left(\frac{\gamma^{1 / 2}}{\sqrt{\bar{\gamma}_{R F} \rho^{2}}} \mid \begin{array}{c}
1 \\
\lambda+1,0
\end{array}\right) d \gamma
$$

By utilizing the integral identity detailed in [33, Eq.(21)], the (23) can be obtained as: 


$$
P_{b_{1}}=\frac{2^{\lambda+1 / 2}}{q^{p \sqrt{2 \pi}} \Gamma(\lambda+1)} G_{3,4}^{2,3}\left(\frac{1}{4 \overline{\gamma \bar{\gamma}_{R F}} \rho^{2} q} \mid \begin{array}{c}
\frac{1}{2}, 1,1-p \\
\frac{\lambda+1}{2}, \frac{\lambda+1}{2}, 0, \frac{1}{2}
\end{array}\right)
$$

Then,

$$
P_{b_{2}}=\frac{1}{\sqrt{2 \pi \sigma^{2} \bar{\gamma}_{F S O}^{2}}} \sum_{k=0}^{\infty} \sum_{j=0}^{2 k}\left(\begin{array}{c}
2 k \\
j
\end{array}\right) \frac{(-1)^{k+j}\left(\bar{\gamma}_{F S O} \mu\right)^{j}}{k !\left(2 \sigma^{2} \bar{\gamma}_{F S O}^{2}\right)^{k}(2 k-j+1)} \int_{0}^{\infty} \gamma^{2 k-j+p} \exp (-q \gamma) d \gamma
$$

By applying the integral identity defined in [30, Eq.(3.326(2))], the (25) can be solved as:

$$
P_{b_{2}}=\frac{1}{\sqrt{2 \pi \sigma^{2} \bar{\gamma}_{F S O}^{2}}} \sum_{k=0}^{\infty} \sum_{j=0}^{2 k}\left(\begin{array}{c}
2 k \\
j
\end{array}\right) \frac{(-1)^{k+j}\left(\bar{\gamma}_{F S O} \mu\right)^{j} \Gamma(2 k-j+p+1)}{k !\left(2 \sigma^{2} \bar{\gamma}_{F S O}^{2}\right)^{k}(2 k-j+1) q^{(2 k-j+p+1)}}
$$

Also,

$$
\begin{array}{r}
P_{b_{3}}=\frac{1}{\Gamma(\lambda+1) \sqrt{2 \pi \sigma^{2} \bar{\gamma}_{F S O}^{2}}} \sum_{k=0}^{\infty} \sum_{j=0}^{2 k}\left(\begin{array}{c}
2 k \\
j
\end{array}\right) \frac{(-1)^{k+j}\left(\bar{\gamma}_{F S O} \mu\right)^{j}}{k !\left(2 \sigma^{2} \bar{\gamma}_{F S O}^{2}\right)^{k}(2 k-j+1)} \\
\quad \times \int_{0}^{\infty} \gamma^{2 k-j+p} \exp (-q \gamma) G_{1,2}^{1,1}\left(\frac{\gamma^{1 / 2}}{\sqrt{\bar{\gamma}_{R F} \rho^{2}}} \mid \begin{array}{c}
1 \\
\lambda+1,0
\end{array}\right) d \gamma
\end{array}
$$

By converting the exponential function to Meijer-G function using the identity detailed in [33, Eq.(11)]. Then, the (27) can be expressed as:

$$
\begin{aligned}
P_{b_{3}}=\frac{1}{\Gamma(\lambda+1) \sqrt{2 \pi \sigma^{2} \bar{\gamma}^{2}}} \sum_{k=0}^{\infty} \sum_{j=0}^{2 k}\left(\begin{array}{c}
2 k \\
j
\end{array}\right) \frac{(-1)^{k+j}(\bar{\gamma} \mu)^{j}}{k !\left(2 \sigma^{2} \bar{\gamma}^{2}\right)^{k}(2 k-j+1)} \\
\quad \times \int_{0}^{\infty} \gamma^{2 k-j+p} G_{0,1}^{1,0}\left(\left.q \gamma\right|_{0} ^{-}\right) G_{1,2}^{1,1}\left(\frac{\gamma^{1 / 2}}{\sqrt{\bar{\gamma}_{R F} \rho^{2}}} \mid \begin{array}{c}
1 \\
\lambda+1,0
\end{array}\right) d \gamma
\end{aligned}
$$

By using the integral identity detailed in [33, Eq.(21)], the (28) can be solved as follows:

$$
\begin{array}{r}
P_{b_{3}}=\frac{2^{\lambda+\frac{1}{2}}}{2 \pi q^{p} \Gamma(\lambda+1) \sqrt{\sigma^{2} \bar{\gamma}_{F S O}^{2}}} \sum_{k=0}^{\infty} \sum_{j=0}^{2 k}\left(\begin{array}{c}
2 k \\
j
\end{array}\right) \frac{(-1)^{k+j}\left(\bar{\gamma}_{F S O} \mu\right)^{j}}{k !\left(2 \sigma^{2} \bar{\gamma}_{F S O}^{2}\right)^{k}(2 k-j+1)} \\
\times G_{3,4}^{2,3}\left(\begin{array}{c|c}
\frac{1}{4 \bar{\gamma}_{R F} \rho^{2} q} & \left.\begin{array}{c}
\frac{1}{2}, 1,2 k-j+p \\
\frac{\lambda+1}{2}, \frac{\lambda+1}{2}, 0, \frac{1}{2}
\end{array}\right)
\end{array}\right.
\end{array}
$$

Therefore, the ABER of the concerned system can be obtained by putting (24), (26), and (29) into (21) as follows: 


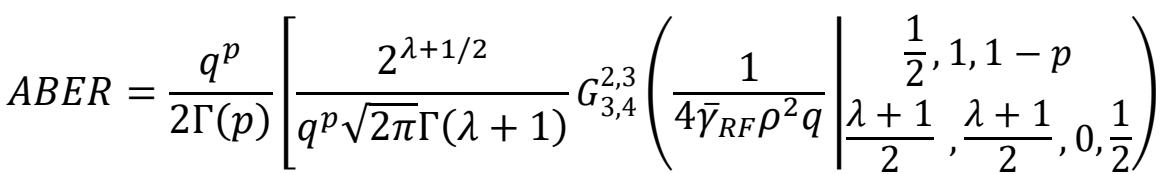

$$
\begin{aligned}
& +\frac{1}{\sqrt{2 \pi \sigma^{2} \bar{\gamma}_{F S O}^{2}}} \sum_{k=0}^{\infty} \sum_{j=0}^{2 k}\left(\begin{array}{c}
2 k \\
j
\end{array}\right) \frac{(-1)^{k+j}\left(\bar{\gamma}_{F S O} \mu\right)^{j} \Gamma(2 k-j+p+1)}{k !\left(2 \sigma^{2} \bar{\gamma}_{F S O}^{2}\right)^{k}(2 k-j+1) q^{(2 k-j+p+1)}} \\
& -\frac{2^{\lambda+1 / 2}}{2 \pi q^{p} \Gamma(\lambda+1) \sqrt{\sigma^{2} \bar{\gamma}_{F S O}^{2}}}
\end{aligned}
$$

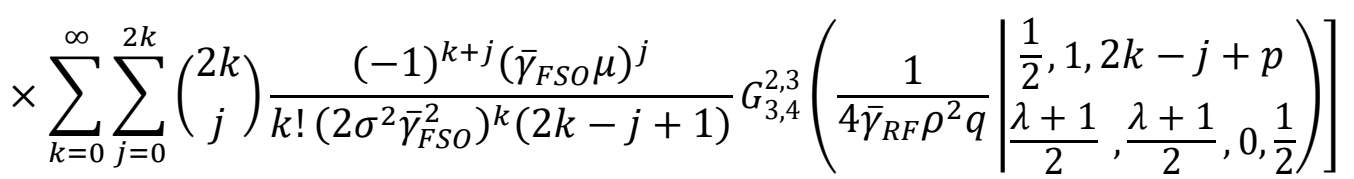

\section{Asymptotic Average Bit Error Rate}

In order to determine the asymptotic analysis for ABER, substitute (19) into (20) and the asymptotic ABER can be expressed as:

$$
A B E R^{\infty}=\frac{q^{p}}{2 \Gamma(p)}\left[P_{b_{2}}+P_{b_{4}}\right]
$$

where

$$
P_{b_{4}}=\frac{1}{\Gamma(\lambda+2)\left(\sqrt{\bar{\gamma}_{R F} \rho^{2}}\right)^{\lambda+1}} \int_{0}^{\infty} \gamma^{p+\frac{\lambda-1}{2}} \exp (-q \gamma) d \gamma
$$

Applying the integral identity detailed in [30, Eq.(3.326(2))], (32) can be solved as:

$$
P_{b_{4}}=\frac{1}{\Gamma(\lambda+2)\left(\sqrt{\bar{\gamma}_{R F} \rho^{2}}\right)^{\lambda+1}} \Gamma\left(p+\frac{\lambda+1}{2}\right) q^{-\left(p+\frac{\lambda+1}{2}\right)}
$$

Thus, the asymptotic ABER for the concerned system can be obtained by putting (26) and (33) into (31) as follows:

$$
\begin{gathered}
A B E R^{\infty}=\frac{q^{p}}{2 \Gamma(p)}\left[\frac{1}{\Gamma(\lambda+2)\left(\sqrt{\bar{\gamma}_{R F} \rho^{2}}\right)^{\lambda+1}} \Gamma\left(p+\frac{\lambda+1}{2}\right) q^{-\left(p+\frac{\lambda+1}{2}\right)}\right. \\
\left.+\frac{1}{\sqrt{2 \pi \sigma^{2} \bar{\gamma}_{F S O}^{2}}} \sum_{k=0}^{\infty} \sum_{j=0}^{2 k}\left(\begin{array}{c}
2 k \\
j
\end{array}\right) \frac{(-1)^{k+j}\left(\bar{\gamma}_{F S O} \mu\right)^{j} \Gamma(2 k-j+p+1)}{k !\left(2 \sigma^{2} \bar{\gamma}_{F S O}^{2}\right)^{k}(2 k-j+1) q^{(2 k-j+p+1)}}\right]
\end{gathered}
$$

\section{Numerical Results and Discussions}

In this section, the numerical results of the outage probability and error rate for the proposed system is presented. Various atmospheric conditions are assumed based on [34] with $\alpha=3.78$ and $\beta=3.78$ for the weak turbulence and $\alpha=2.04$ and $\beta=1.10$ for the strong turbulence. Except otherwise stated, $\bar{\gamma}_{t h}=5 \mathrm{~dB}, \mathrm{~m}=2, w_{z}=1.2, \sigma_{\beta}=0.5 \mathrm{mrad}, \sigma_{\theta}=1 \mathrm{mrad}$, $L_{1}^{F S O}=L_{2}^{F S O}=50 \mathrm{~m}$ and $L_{1}^{R F}=L_{2}^{R F}=20 \mathrm{~m}$. 
The impact of number of reflecting elements on the RIS-RF link under the strong atmospheric turbulence is illustrated in figure 2. It can be observed that the system outage probability performance significantly improves as the $N_{R F}$ increases and large value of $N_{R F}$ offers better performance. From the results, it can also be deduced that the analytical results match with the simulation results perfectly.

The effect of atmospheric turbulence and the number of reflecting elements in RIS-FSO link on the concerned system is presented in figure 3 . The results demonstrate that the atmospheric turbulence from weak to strong level significantly deteriorate the system outage performance. Under both conditions, the increase in the number of $N_{F S O}$ offers the system better performance. Further, the results depict that there is a perfect agreement between the simulation results and the analytical results which indicate the accuracy of the derived outage probability expression.

The outage performance of the proposed system for different values of threshold SNR is presented in figure 4 under different turbulence conditions. The results illustrate that the increase in threshold values degrades the system performance with lower value offers better performance. Similar to figure 3, it is established that the atmospheric turbulence great influence on the system performance. The results also show that the analytical results are consistent with simulation results. In addition, at high SNRs, the asymptotic results are perfectly matched with analytical results which confirm the correctness of the analytical expression.

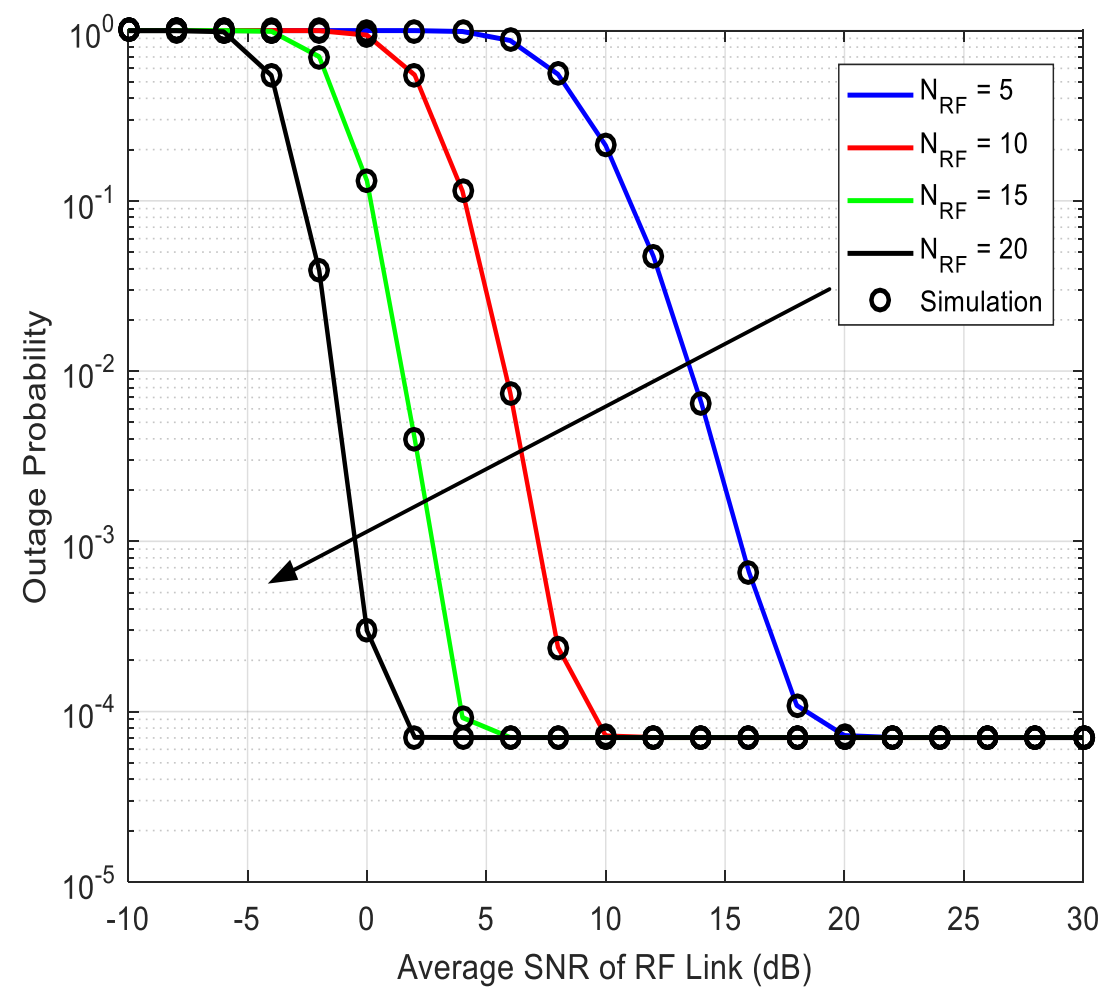

Figure 2: Outage probability performance of the system at $\bar{\gamma}_{F S O}=35 \mathrm{~dB}$ under strong turbulence condition for different values of $N_{R F}$ at $N_{F S O}=50$ 


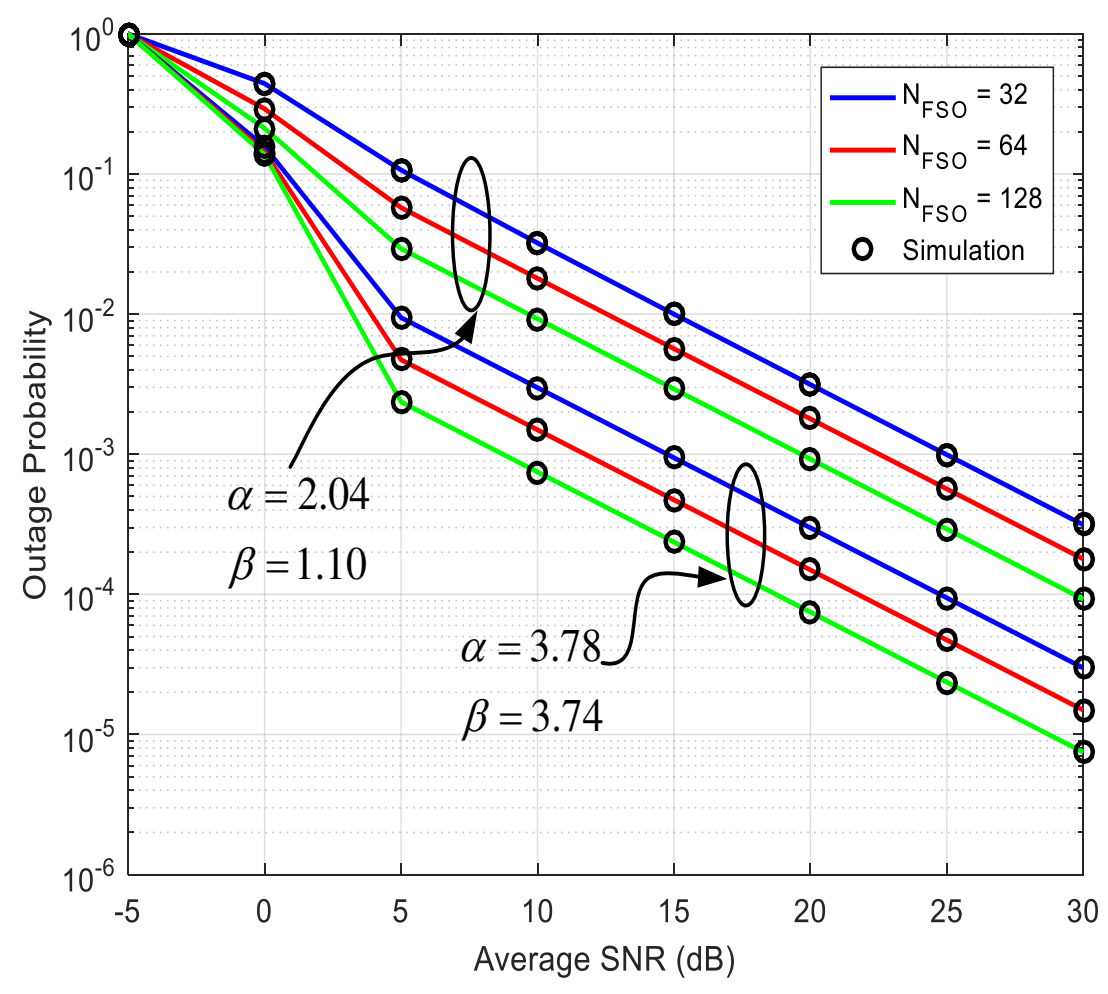

Figure 3: Impact of atmospheric turbulence conditions on the system performance under different values of $N_{F S O}$ at $N_{R F}=15$

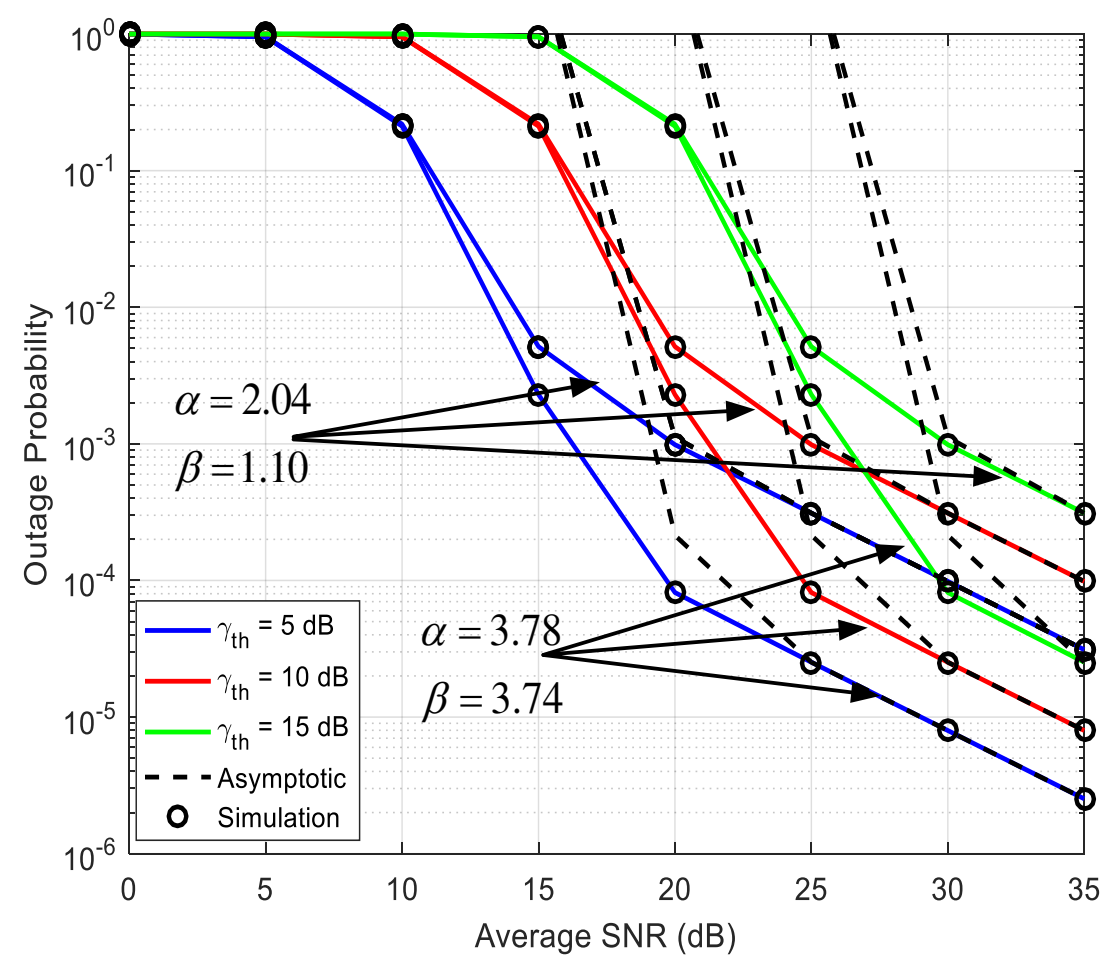

Figure 4: Effect of atmospheric turbulence on the system performance under different values of $\gamma_{t h}$ at $N_{F S O}=120$ and $N_{R F}=5$ 
In figure 5 , the influence of fading $m$ parameter for the RF link on the outage performance under different values of $N_{R F}$ is depicted. The results show that the increase in m leads to improvement in quality of RF link fading and hence offers the system better performance. Similar as in figure 1, the increase in $N_{R F}$ significantly enhance the system performance for different values of $m$ parameters. Further, the analytical results collaborate with simulation results which validate the use of the derived expression.

Moreover, the impact of atmospheric turbulence on the system error rate is presented in figure 6 under different values of $N_{F S O}$. It can be deduced from the results that the increase in the level of atmospheric turbulence from weak to strong significantly deteriorate the system error performance. However, under the same atmospheric conditions, the increase in $N_{F S O}$ enhances the system performance. Also, the results indicate that the asymptotic results converge to the analytical results at high SNRs which prove the use of the derived error rate expression.

The influence of number of reflecting elements $N_{R F}$ in RIS-RF link on the system error rate is presented in figure 7 under different modulation schemes. It is clearly shown that the BPSK outperforms the DPSK under the same system conditions. Further, the increase in the $N_{R F}$ significantly offer the system better performance.

The impact of pointing error under the beam width $w_{z}$ condition on the system performance is illustrated in figure 8 for different fading $m$ parameter. It can be deduced from the results that the increase in $m$ enhances the quality of link and thus leads to better system performance. Also, it can be observed that as the $w_{z}$ decreases, the better the system performance since high receive power is incur in narrow beam width. However, the narrow beam width is highly prone to loss of line of sight due to misalignment effect.

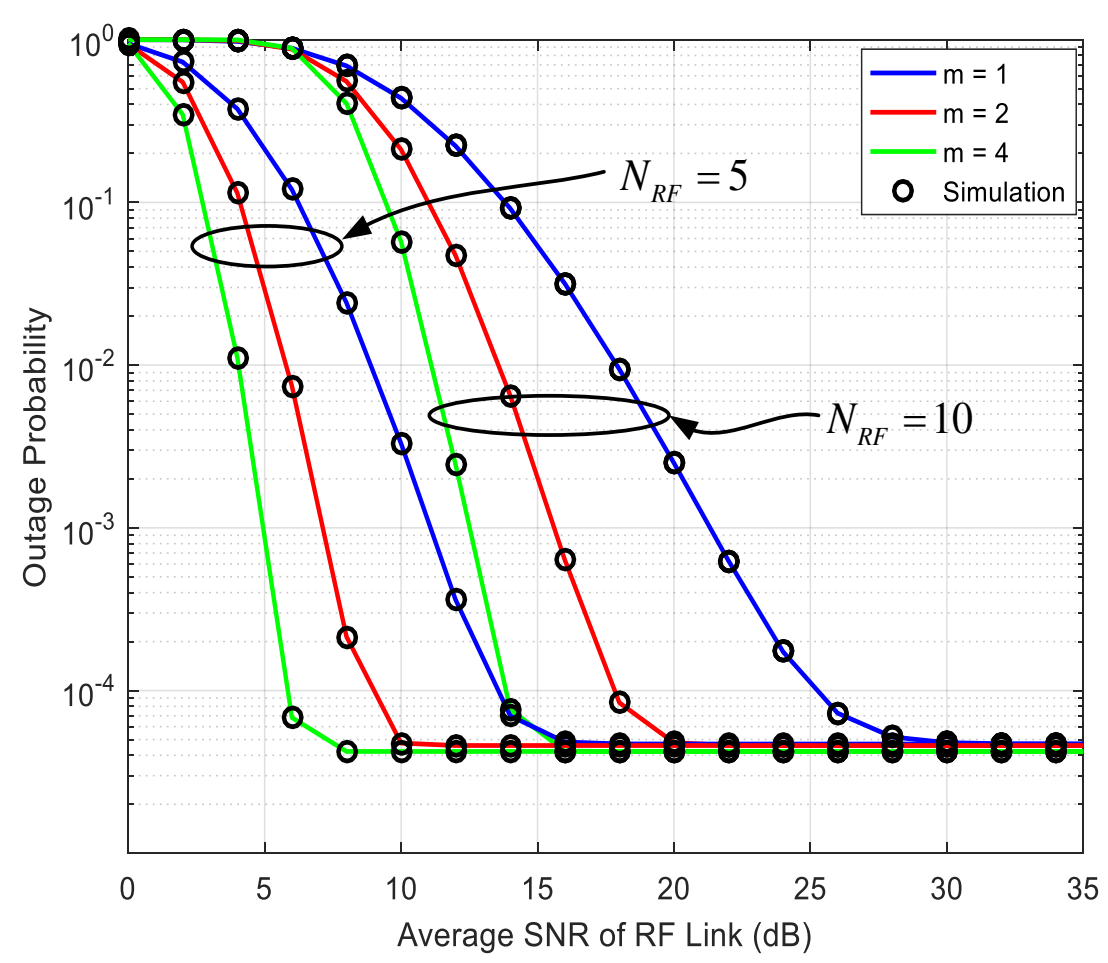

Figure 5: Influence of $m$ fading parameter on the system performance under strong turbulence condition at different values of $N_{R F}$ and $N_{F S O}=80$ 


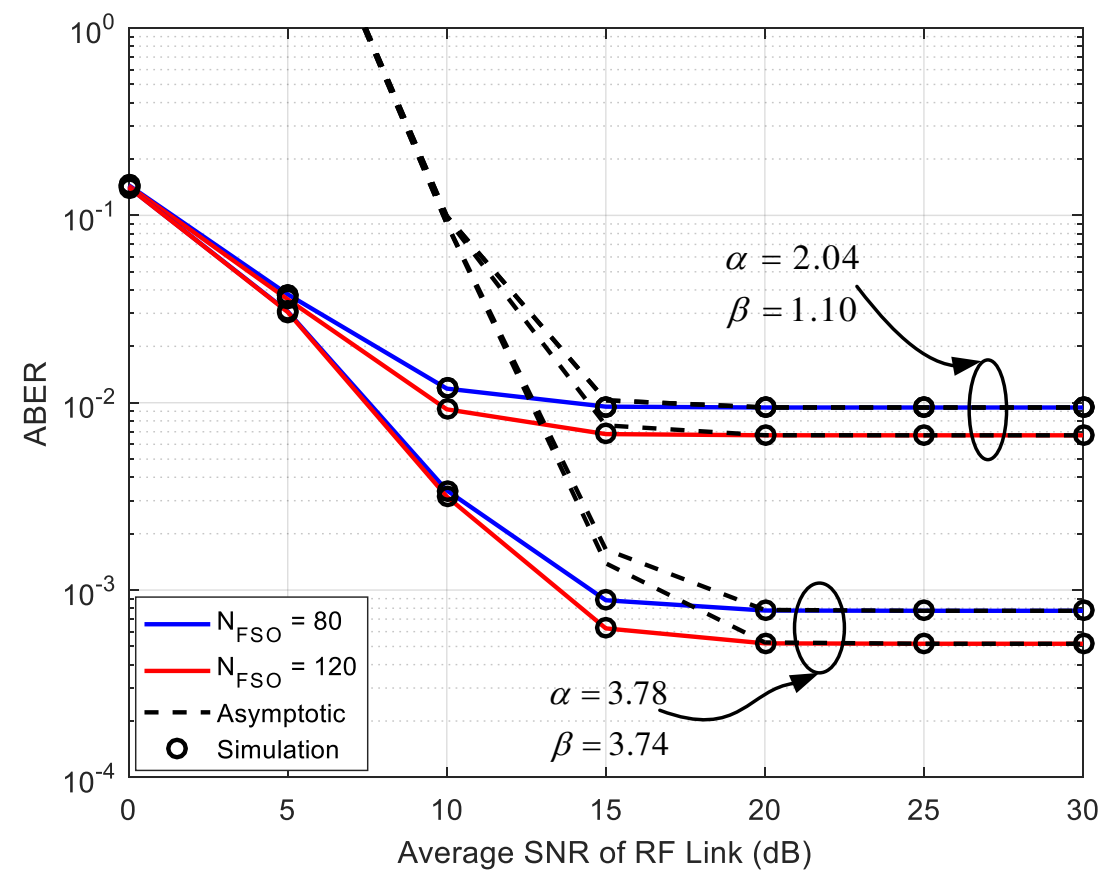

Figure 6: Error rate performance of the system at different atmospheric condition and values of $N_{F S O}$ under BPSK modulation for $N_{R F}=5$ and $\bar{\gamma}_{F S O}=10 \mathrm{~dB}$

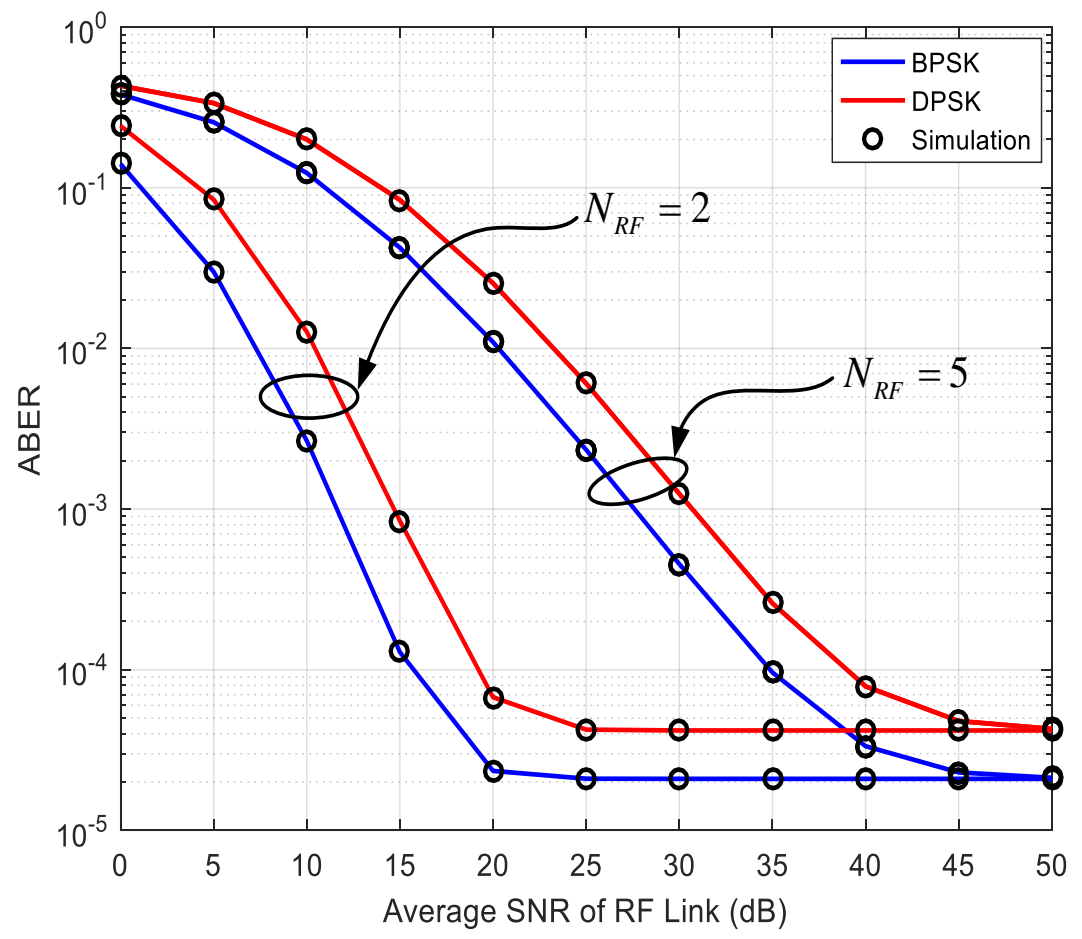

Figure 7: Error rate performance of the system for different modulation schemes under strong turbulence conditions at different values of $N_{R F}$ for $m=1$ and $\bar{\gamma}_{F S O}=35 \mathrm{~dB}$ 


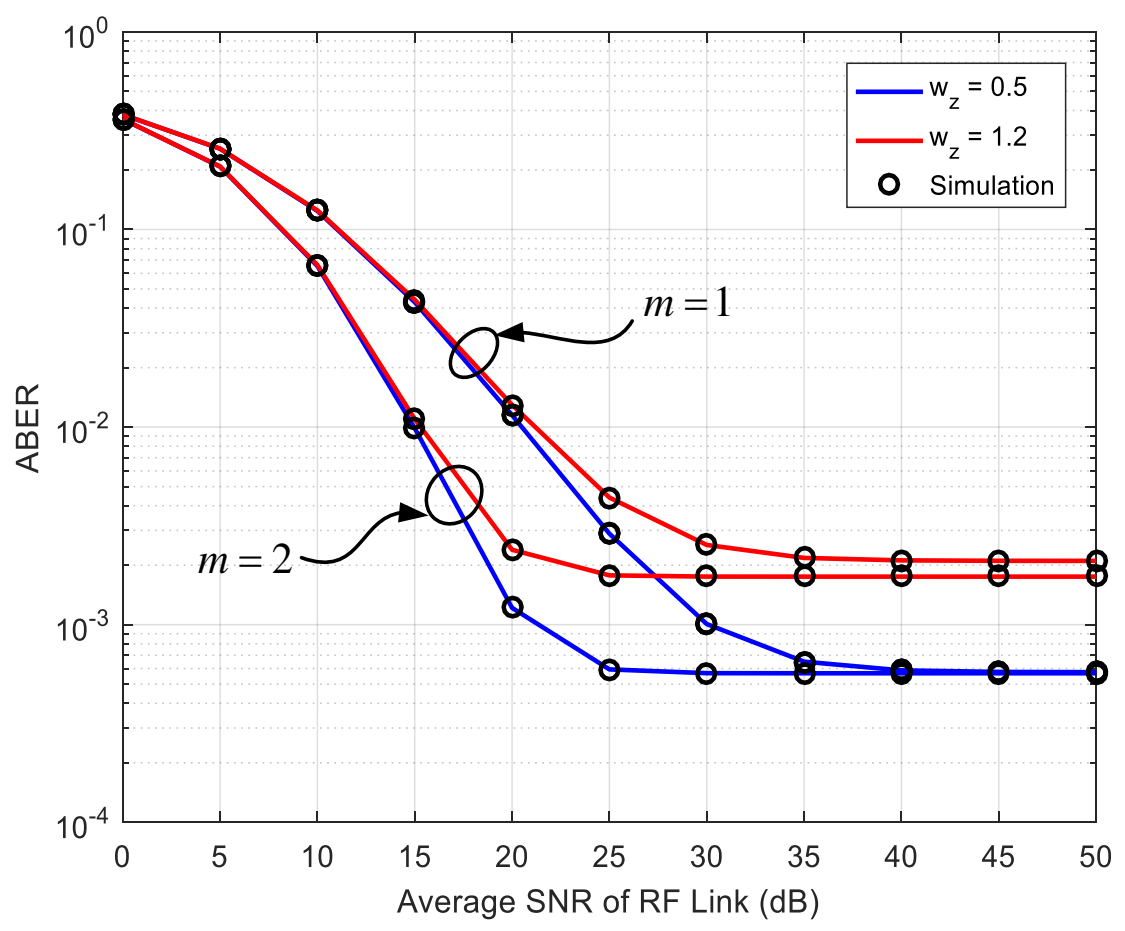

Figure 8: Effect of beam width $w_{Z}$ on the system error rate at different values of $m$ parameters under BPSK modulation and strong turbulence condition when $N_{R F}=5, N_{F S O}=$ 80 , and $\bar{\gamma}_{F S O}=15 d B$

\section{Conclusion}

The performance of RIS in a dual-hop DF relay empowered asymmetric RF/FSO systems is investigated in this paper. The closed-form expressions of the system outage probability and average error rate are derived. In order to gain more insight about the system behaviour, the asymptotic expressions are obtained for the concerned system. Moreover, the results show that the analytical results collaborate well with the simulation results. It is also illustrated that the number of reflecting elements in both RIS-RF and RIS-FSO significantly enhance the system performance. Further, the increase in the channel $m$-fading parameters on the RF offers the system better performance. It is also deduced from the results that the pointing errors and atmospheric turbulence significantly degrade the system performance.

Funding: No funding was received for conducting this study

Data Availability Statement: The Manuscript has no associated data

Conflicts of Interest: The authors declare no conflict of interest

\section{Reference}

[1] M. I. Petkovic, "Performance analysis of mixed RF/FSO systems," in $201523 \mathrm{rd}$ Telecommunications Forum Telfor (TELFOR), 2015, pp. 293-300: IEEE.

[2] I. Trigui, S. Affes, A. M. Salhab, and M.-S. Alouini, "Multi-user mixed FSO-RF systems with aperture selection under poisson field interference," IEEE Access, vol. 7, pp. 73764-73781, 2019. 
[3] K. O. Odeyemi, P. A. Owolawi, and V. M. Srivastava, "Performance analysis of free space optical system with spatial modulation and diversity combiners over the Gamma Gamma atmospheric turbulence," Optics Communications, vol. 382, pp. 205-211, 2017.

[4] K. O. Odeyemi, P. A. Owolawi, and V. M. Srivastava, "Optical spatial modulation over gamma-gamma turbulence and pointing error induced fading channels," Optik, vol. 147, pp. 214-223, 2017.

[5] H. Liang, Y. Li, M. Miao, C. Gao, and X. Li, "Unified Performance Analysis of MIMO Mixed RF/FSO Relaying System," Applied Sciences, vol. 11, no. 7, p. 3054, 2021.

[6] E. Soleimani-Nasab and M. Uysal, "Generalized performance analysis of mixed RF/FSO cooperative systems," IEEE Transactions on Wireless Communications, vol. 15, no. 1, pp. 714-727, 2015.

[7] N. H. Juel et al., "Secrecy Performance Analysis of Mixed $\alpha-\mu$ and Exponentiated Weibull RF-FSO Cooperative Relaying System," IEEE Access, 2021.

[8] H. Lei et al., "On secure mixed RF-FSO systems with TAS and imperfect CSI," IEEE Transactions on Communications, vol. 68, no. 7, pp. 4461-4475, 2020.

[9] N. Varshney and P. Puri, "Performance analysis of decode-and-forward-based mixed MIMO-RF/FSO cooperative systems with source mobility and imperfect CSI," Journal of Lightwave Technology, vol. 35, no. 11, pp. 2070-2077, 2017.

[10] L. Yang, F. Meng, Q. Wu, D. B. da Costa, and M.-S. Alouini, "Accurate closed-form approximations to channel distributions of RIS-aided wireless systems," IEEE Wireless Communications Letters, vol. 9, no. 11, pp. 1985-1989, 2020.

[11] M. H. Samuh and A. M. Salhab, "Performance analysis of reconfigurable intelligent surfaces over Nakagami-m fading channels," arXiv preprint arXiv:.07841, 2020.

[12] L. Yang, F. Meng, J. Zhang, M. O. Hasna, and M. Di Renzo, "On the performance of RIS-assisted dual-hop UAV communication systems," IEEE Transactions on Vehicular Technology, vol. 69, no. 9, pp. 10385-10390, 2020.

[13] L. Yang, W. Guo, D. B. da Costa, and M.-S. Alouini, "Free-Space Optical Communication With Reconfigurable Intelligent Surfaces," arXiv preprint arXiv:.00547, 2020.

[14] A. R. Ndjiongue, T. Ngatched, O. A. Dobre, A. G. Armada, and H. Haas, "Performance Analysis of RIS-Based nT-FSO Link Over GG Turbulence With Pointing Errors," arXiv preprint arXiv:.03654, 2021.

[15] A. U. Makarfi, K. M. Rabie, O. Kaiwartya, O. S. Badarneh, X. Li, and R. Kharel, "Reconfigurable intelligent surface enabled IoT networks in generalized fading channels," in ICC 2020-2020 IEEE International Conference on Communications (ICC), 2020, pp. 1-6: IEEE.

[16] A. E. Canbilen, E. Basar, and S. S. Ikki, "Reconfigurable intelligent surface-assisted space shift keying," IEEE Wireless Communications Letters, vol. 9, no. 9, pp. 14951499, 2020.

[17] K. O. Odeyemi, P. A. Owolawi, and O. O. Olakanmi, "On the Performance of Reconfigurable Intelligent Surface Aided Power Line Communication System under Different Relay Transmission Protocols," Prog. Electromagn. Res. C, vol. 111, pp. 119133, 2021.

[18] S. Li, L. Yang, D. B. da Costa, M. Di Renzo, and M.-S. Alouini, "On the Performance of RIS-Assisted Dual-Hop Mixed RF-UWOC Systems," IEEE Transactions on Cognitive Communications Networking, 2021.

[19] K. O. Odeyemi, P. A. Owolawi, and O. O. Olakanmi, "Performance Analysis of Reconfigurable Intelligent Surface Assisted Underwater Optical Communication System," Progress In Electromagnetics Research, vol. 98, pp. 101-111, 2020. 
[20] Y. Ai, F. A. de Figueiredo, L. Kong, M. Cheffena, S. Chatzinotas, and B. Ottersten, "Secure vehicular communications through reconfigurable intelligent surfaces," arXiv preprint arXiv:.14899, 2020.

[21] A. U. Makarfi, K. M. Rabie, O. Kaiwartya, X. Li, and R. Kharel, "Physical layer security in vehicular networks with reconfigurable intelligent surfaces," in 2020 IEEE 91st Vehicular Technology Conference (VTC2020-Spring), 2020, pp. 1-6: IEEE.

[22] L. Yang, Y. Yang, D. B. da Costa, and I. Trigui, "Performance Analysis of an Interference-Limited RIS-Aided Network," arXiv preprint arXiv:.07479, 2020.

[23] K. Odeyemi, P. Owolawi, and O. Olakanmi, "Reconfigurable Intelligent Surface in Wireless-Powered Interference-Limited Communication Networks," Symmetry, vol. 13, no. 6, p. 960, 2021.

[24] A. M. Salhab and L. Yang, "Mixing RIS-Assisted Sources with FSO Link," arXiv preprint arXiv:.05612, 2020.

[25] L. Yang, W. Guo, and I. S. Ansari, "Mixed dual-hop FSO-RF communication systems through reconfigurable intelligent surface," IEEE Communications Letters, vol. 24, no. 7, pp. 1558-1562, 2020.

[26] I. S. Ansari, F. Yilmaz, and M.-S. Alouini, "Impact of pointing errors on the performance of mixed RF/FSO dual-hop transmission systems," IEEE Wireless Communications Letters, vol. 2, no. 3, pp. 351-354, 2013.

[27] A. Prudnikov, Y. A. Brychkov, and O. Marichev, Integrals and series Volume 3: More special functions. Taylor and Francis, Oxford, UK, 2003.

[28] H. Wang et al., "Performance of wireless optical communication with reconfigurable intelligent surfaces and random obstacles," arXiv preprint arXiv:.05715, 2020.

[29] J. Proakis, "Digital communications, new york: Mcgrawhill," ed: Inc, 2001.

[30] I. S. Gradshteyn and I. M. Ryzhik, Table of integrals, series, and products. Academic press, 2014.

[31] L. Yang, M.-S. Alouini, and I. S. Ansari, "Asymptotic performance analysis of twoway relaying FSO networks with nonzero boresight pointing errors over doublegeneralized gamma fading channels," IEEE Transactions on Vehicular Technology, vol. 67, no. 8, pp. 7800-7805, 2018.

[32] S. Li, L. Yang, D. B. Da Costa, and S. Yu, "Performance analysis of UAV-based mixed RF-UWOC transmission systems," IEEE Transactions on Communications, 2021.

[33] V. Adamchik and O. Marichev, "The algorithm for calculating integrals of hypergeometric type functions and its realization in REDUCE system," in Proceedings of the international symposium on Symbolic and algebraic computation, 1990, pp. 212224.

[34] K. O. Odeyemi, P. A. Owolawi, and V. M. Srivastava, "Performance analysis of decode-and-forward dual-hop optical spatial modulation with diversity combiner over atmospheric turbulence," Optics Communications, vol. 402, pp. 242-251, 2017. 